\title{
Comparison of monitoring performance of bioreactance versus esophageal Doppler in pediatric patients
}

\author{
Clément Dubost, Adrien Bouglé 1 , Calliope Hallynck², Matthieu Le Dorze ${ }^{3}$, Philippe Roulleau ${ }^{4}$, \\ Catherine Baujard ${ }^{4}$, Dan Benhamou ${ }^{4}$
}

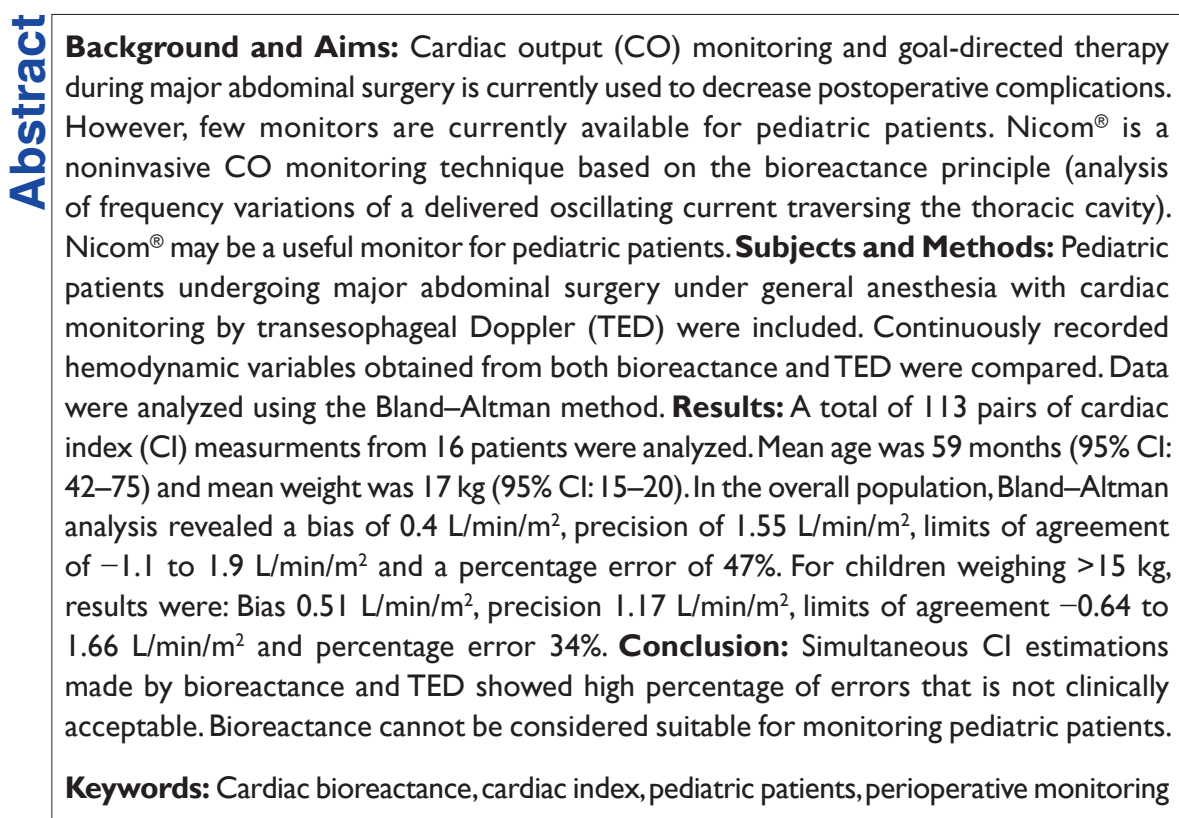

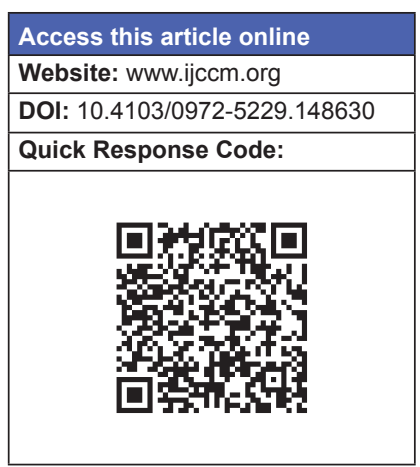

\section{Introduction}

Hemodynamic monitoring during major surgery is critical to guide fluid resuscitation. Recently, two meta-analyses have confirmed that perioperative goal-directed fluid therapy improves postoperative outcome in adults. ${ }^{[1,2]}$ Among the various techniques aimed at measuring cardiac output (CO), transesophageal Doppler (TED) monitoring has received the strongest

From:

Départment of Anesthésie-Réanimation, Hôpital Bicêtre, Le KremlinBicêtre, France, ${ }^{1}$ Surgical Intensive Care Unit, Institut of Cardiology, Groupe Hospitalier Pitié-Salpêtrière, Réanimation Médicale Polyvalente,

${ }^{2}$ Départment of Anesthésie-Réanimation, Hôpital Saint-Antoine, ${ }^{3}$ Départment of Anesthésie-Réanimation, Hôpital Lariboisière, Paris, ${ }^{4}$ Départment Anesthésie-Réanimation, Hôpital Bicêtre, Le Kremlin-Bicêtre, France

\section{Correspondence:}

Dr. Clement Dubost, Consultant in Anesthesiology and Intensive Care Medicine, Begin Military Hospital, 69 Avenue de Paris, 94163 Saint-Mande,

France. E-mail: clement.dubost@ hotmail.fr validation, ${ }^{[3]}$ but other monitors could also be used and include pulmonary artery catheter (PAC), ${ }^{[4]}$ arterial pressure waveform analysis ${ }^{[5]}$ or pulse pressure variation. ${ }^{[6]}$ In the pediatric population, data are lacking, but extrapolation seems acceptable, especially in the case of major surgery.

The gold standard for hemodynamic monitoring remains the PAC. However, the permanent search to be less and less invasive has led to the development of non or less invasive $\mathrm{CO}$ monitors. ${ }^{[7]}$ It is an exciting area of research but unfortunately still few less invasive devices have been well-validated ${ }^{[8]}$ and even fewer noninvasive hemodynamic monitors are currently suitable for pediatric patients. TED is available with dedicated pediatric probes and monitors. It has proved to have an acceptable monitoring performance in children and is 
a promising technique. ${ }^{[9,10]}$ However, TED has several limitations, including electrical interactions with surgical electric cautery and probe misplacement.

Transthoracic bioreactance is a recent technique based on analysis of the frequency variations of a delivered oscillating current traversing the thoracic cavity. ${ }^{[11]}$ Contrary to bioimpedance, in which only variations in transthoracic voltage were studied, bioreactance is based on the analysis of intrabeat variations in voltage phase shifts. ${ }^{[12]}$ Studies have validated this technique in adults with comparison to PAC ${ }^{[13]}$ and transpulmonary thermodilution. ${ }^{[14]}$ A study of bioreactance in children has been published, but there was no comparison with a reference technology. ${ }^{[15]}$ The authors concluded that the cardiac index (CI) measurement obtained by bioreactance varies with age and weight of the patient but was lower than the normal range. Two other studies have recently been published, one focusing on neonate ${ }^{[16]}$ and the other using a piglet model. ${ }^{[17]}$ These two studies have concluded that values obtained from bioreactance were consistently lower than that obtained with other techniques.

The aim of this study was to compare CI monitoring performance of bioreactance (Nicom ${ }^{\circledR}$, Cheetah Medical, Newton Center, MA 02459, USA) using TED (CardioQP ${ }^{\circledR}$, Deltex Medical, Chichester, UK) as a standard method in children undergoing major abdominal surgery.

\section{Materials and Methods}

The study protocol was accepted by the ethics committee Paris Ile de France III under the number S.C. 2896 on 7 June 2010 . As the protocol did not include any change from usual care, the ethics committee waived the parent/tutor approval and only requested the delivery of written information.

\section{Study population}

From September 2010 to March 2011, all pediatric patients in whom TED monitoring was used intraoperatively and who were scheduled for elective major abdominal surgery were included. TED is currently systematically used for major abdominal surgery in pediatrics by all the anesthetists of the team. There was no modification of care due to the protocol. We performed a power analysis using an alpha-risk of $5 \%$ and a power of $80 \%$. To detect a difference between the two methods of $50 \% 10$ subjects were necessary and 16 for a $30 \%$ difference.

\section{Monitoring and follow-up of the patients}

According to the manufacturer, the bioreactance monitor is suitable for patients ranging from newborns to adults. For the two devices, anthropometric data were entered in the device before starting the exam. Bioreactance's electrodes were placed before beginning of anesthesia in the patient's back. The survey started after induction of anesthesia because the TED probe was inserted only in anesthetized patients. Values were manually recorded by an independent operator. Screens of both devices were frozen to ensure that measurements were taken simultaneously. Automatic data collection was available for $\mathrm{Nicom}^{\circledR}$ but not for the TED. This implies that after the end of the surgery, TED's values that have not been manually collected were lost. The values recorded were: Sex, age, weight, height, respiratory end tidal volume, respiratory rate, positive end expiratory pressure value (if any), vasoactive drug infusion rate, heart rate, blood pressure, pulse pressure variation (if available), and for both monitors: $\mathrm{CI}$ and stroke volume indexed (SVI). At least 5 min between two consecutive measurements were deemed mandatory and no $>10$ observations per patient were recorded.

A change in CI was defined as a $\pm 15 \%$ variation compared with the immediately obtained previous measurement.

\section{Statistical analysis}

Owing to the small sample size, data were considered to have a nonnormal distribution and nonparametric tests were used. Continuous data are expressed as median with 95\% confidence interval (95\% CI). The Spearman's correlation coefficient and the Bland-Altman method were used to compare results obtained with the two monitors. ${ }^{[18]}$ Recently published recommendations on how to track changes in CO were followed. ${ }^{[19]}$ Precision, coefficient of variation and percentage of errors were calculated consequently. Based on the conclusion of Peyton and Chong, we defined an acceptable percentage error as $<45 \% .{ }^{\left[{ }^{[8]}\right.}$ Because heart rate is a major component of $\mathrm{CI}$ in children, comparison of the SVI obtained from the two methods was also performed. Value of $P<0.05$ was considered significant. Statistical analysis was performed using Prism ${ }^{\circledR} 4.00$ for Mac (Inc., GraphPad Software, San Diego California USA, http://www. graphpad.com).

\section{Results}

During the study period, 16 patients were included for a total amount of 113 pairs of measurements. Baseline data are presented in Table 1. During the study period, a total of 30 patients has been electively operated for major abdominal surgery, meaning that we included $53 \%$ of the cases. 
Cardiac output measurement using bioreactance was found to be not feasible in children weighing $<10 \mathrm{~kg}$ because electrodes overlapped (too large size as regards to the chest size). As a consequence, no patient weighing $<10 \mathrm{~kg}$ has been included. For patients weighing $>10 \mathrm{~kg}$, values from both techniques were recorded without any problems. The quality of the signal was good for both monitors and loss of signal occurred when surgical electric cautery was used but to the same extent for both devices.

Between 3 and 10 pairs of measurements were obtained for each patient.

\section{Cardiac index comparisons}

The Spearman's correlation coefficient between bioreactance and TED was $0.433(P<0.0001)$ [Figure 1]. The mean values of SVI for bioreactance and TED were 2.89 and $3.29 \mathrm{~mL} / \mathrm{min} / \mathrm{m}^{2}$ respectively. The mean value of heart rate was 108 beats/min. Bland-Altman analysis revealed a bias of $0.4 \mathrm{~L} / \mathrm{min} / \mathrm{m}^{2}$, precision of $1.55 \mathrm{~L} / \mathrm{min} / \mathrm{m}^{2}$, limits of agreement of -1.1 to $1.9 \mathrm{~L} / \mathrm{min} / \mathrm{m}^{2}$ and a percentage error of $47 \%$ [Figure 2]. The coefficients of variations for the bioreactance and the TED were $27 \%$ and $24 \%$, respectively.

\section{Stroke volume indexed comparisons}

The Spearman's correlation coefficient between bioreactance and TED was $0.47(P<0.0001)$ [Figure 3]. The mean values of stroke SVI for bioreactance and TED were 27.54 and $27.32 \mathrm{~mL} / \mathrm{m}^{2}$ respectively. Bland-Altman analysis revealed a bias of $0.22 \mathrm{~mL} / \mathrm{m}^{2}$, precision of $22 \mathrm{~mL} / \mathrm{m}^{2}$, limits of agreement of -21.79 to $21.35 \mathrm{~mL} / \mathrm{m}^{2}$ and a percentage error of $81 \%$ [Figure 4]. The coefficients of variations for the bioreactance and the TED were $35 \%$ and $49 \%$, respectively.

\begin{tabular}{lc}
\hline Table I: Baseline data of the I 6 children included \\
\hline Age (months) & $59(42-75)$ \\
Weight $(\mathrm{kg})$ & $17(15-20)$ \\
Tidal volume $(\mathrm{mL})$ & $164(133-195)$ \\
Respiratory rate & $22(19-25)$ \\
PEEP $\left(\mathrm{cmH}_{2} \mathrm{O}\right)$ & $3(\mathrm{I}-4)$ \\
\hline
\end{tabular}

To check if the accuracy was sensible to the child's weight, separate analysis was performed for children weighing >15, 17, and $19 \mathrm{~kg}$ [Table 2, Figures 5 and 6].

We compared directional changes of CI (unchanged, increasing or decreasing trend) assessed by the two techniques. In the whole population, variations occurred, in the same way, in 61 out of 97 cases (63\%). When focusing on the population with weight above $15 \mathrm{~kg}$, the rate was 44 out of $67(66 \%)$.

\section{Discussion}

We found that in this pediatric population, the coefficient of correlation between values of CI obtained by bioreactance and TED monitoring was poor. Bland-Altman analysis revealed acceptable limits of agreement, ${ }^{[8,18]}$ but a high percentage of error $(47 \%)$. When limiting the analysis to children weighing $>15 \mathrm{~kg}$, the percentage of error was less than for the all group (34\%) but the variations of CI occurred in the same way in only $66 \%$ of the cases.

Two technical limitations were observed. First, CI monitoring using bioreactance appears feasible only in pediatric patients weighing $>10 \mathrm{~kg}$. Indeed, bioreactance measurement is made through four pairs of electrodes (two at the top and two at the bottom of the chest) and in this population of small children electrodes overlap. Second, we were not able to automatically collect the data because the TED monitor we used did not have a memory.

When considering the overall population studied, Bland-Altman analysis revealed acceptable limits of agreement, ${ }^{[8,18]}$ but a high percentage of error $(47 \%)$. When considering children $\geq 15 \mathrm{~kg}$, the percentage of error was $34 \%$ and when considering only patients weighing $>17$ or $>19 \mathrm{~kg}$ it was even closer to the traditional threshold of validity (i.e. 31 and $32 \%$, respectively). CI changed in the same direction with both monitoring devices in only $61 / 97$ (63\%) patients in the overall population and in $44 / 67(66 \%)$ in the population of children weighing $>15 \mathrm{~kg}$. This value could be improved by taking into account the respective latency of each device. The TED is reported to have a very short latency; ${ }^{[20]}$ as for the $\mathrm{Nicom}^{\circledR}$, its latency is at least $30 \mathrm{~s}$ (based on the

Table 2: Bland-Altman analysis and its relation to the weight of the subgroup considered

\begin{tabular}{|c|c|c|c|c|c|c|c|}
\hline $\begin{array}{l}\text { Weight } \\
\text { (kg) }\end{array}$ & $\begin{array}{l}\text { Number } \\
\text { of patient }\end{array}$ & $\begin{array}{l}\text { Number of } \\
\text { observations }\end{array}$ & $\begin{array}{l}\text { Spearman } \\
\text { coefficient }\end{array}$ & $\begin{array}{c}\text { Bias } \\
\mathrm{L} / \mathrm{min} / \mathrm{m}^{2}\end{array}$ & $\begin{array}{l}\text { Precision } \\
\mathrm{L} / \mathrm{min} / \mathrm{m}^{2}\end{array}$ & $\begin{array}{c}\text { Limits agreement } \\
\mathrm{L} / \mathrm{min} / \mathrm{m}^{2}\end{array}$ & $\begin{array}{c}\text { Percentage } \\
\text { error }\end{array}$ \\
\hline$>15$ & 12 & 79 & 0.56 & 0.51 & 1.17 & -0.64 to 1.66 & 34 \\
\hline$>17$ & 7 & 47 & 0.69 & 0.64 & I.II & -0.45 to 1.73 & 31 \\
\hline$>19$ & 6 & 41 & 0.74 & 0.58 & 1.13 & -0.53 to 1.69 & 32 \\
\hline
\end{tabular}




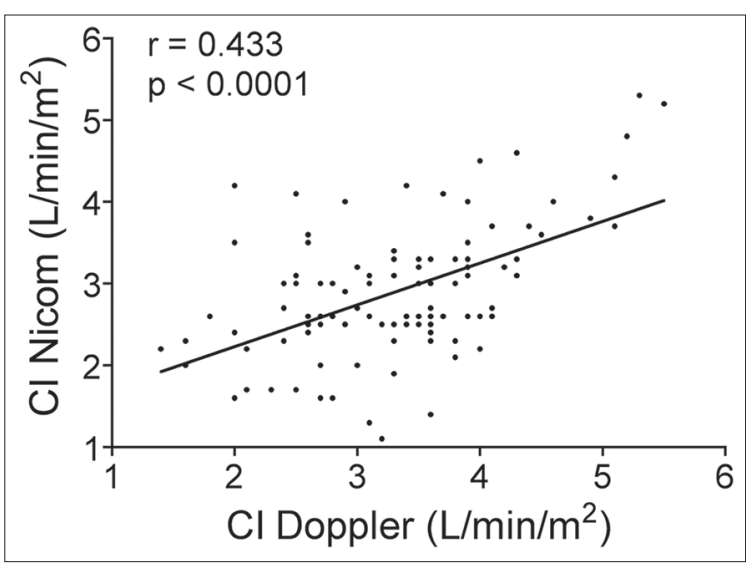

Figure I: Spearman coefficient correlation of cardiac index between transesophageal Doppler and bioreactance in the global population

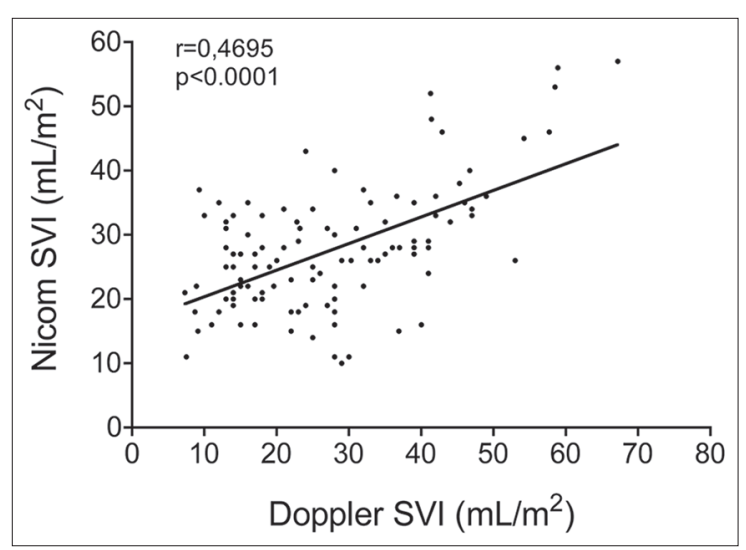

Figure 3: Spearman coefficient correlation of stroke volume indexed between transesophageal Doppler and bioreactance in the global population

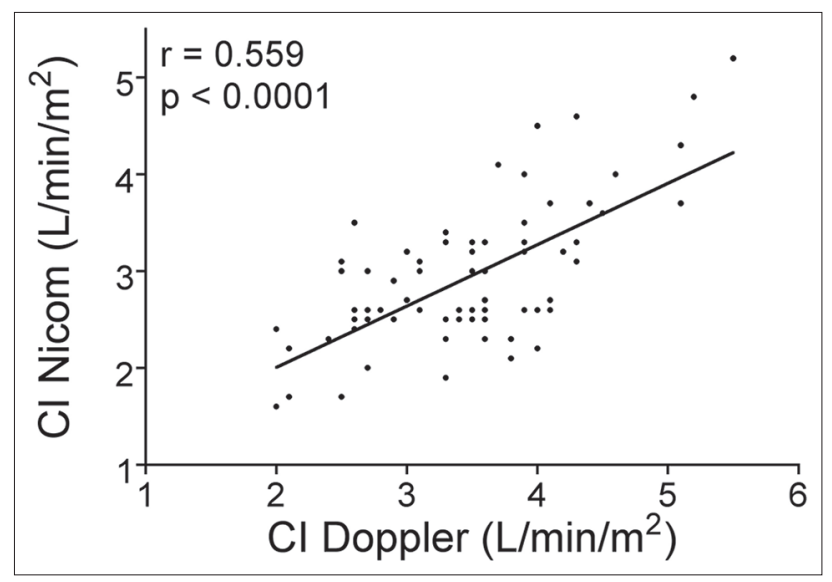

Figure 5: Spearman coefficient correlation between transesophageal Doppler and bioreactance in the population of children weighing $>15 \mathrm{~kg}$

minimum sample interval value). Our experience was that bioreactance changes occurred faster than with TED.

Contrary to the results recently obtained by Weisz et al. in nine neonates, we were not able to monitor patients weighing $<10 \mathrm{~kg}$. ${ }^{[16]}$ This may be explained

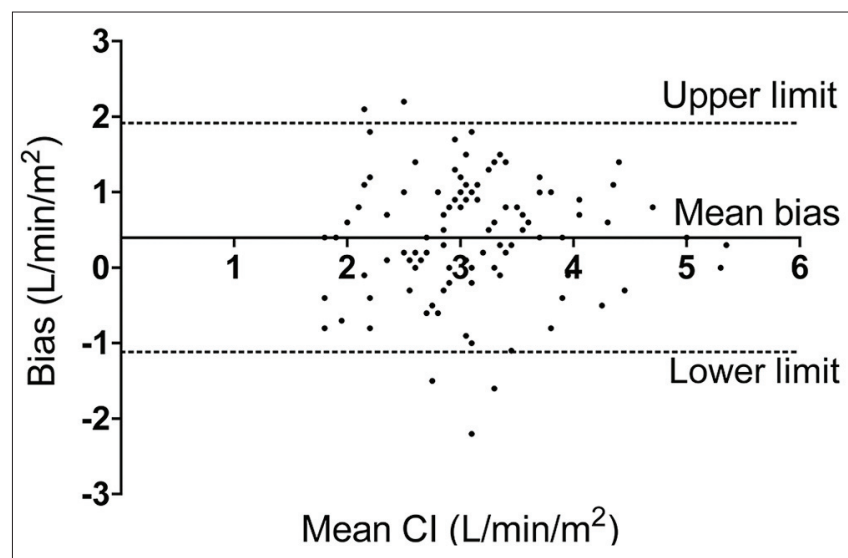

Figure 2: Bland-Altman analysis of cardiac index between transesophageal Doppler and bioreactance in the global population

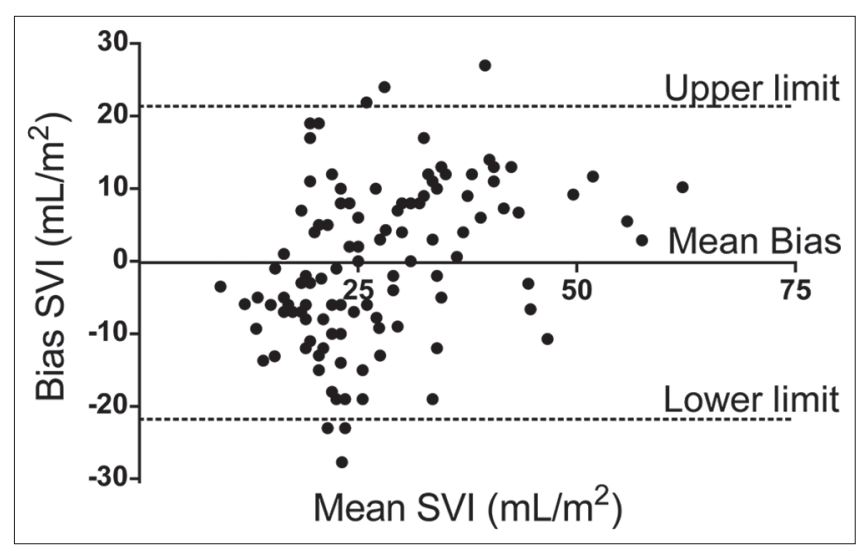

Figure 4: Bland-Altman analysis of stroke volume indexed between transesophageal Doppler and bioreactance in the global population

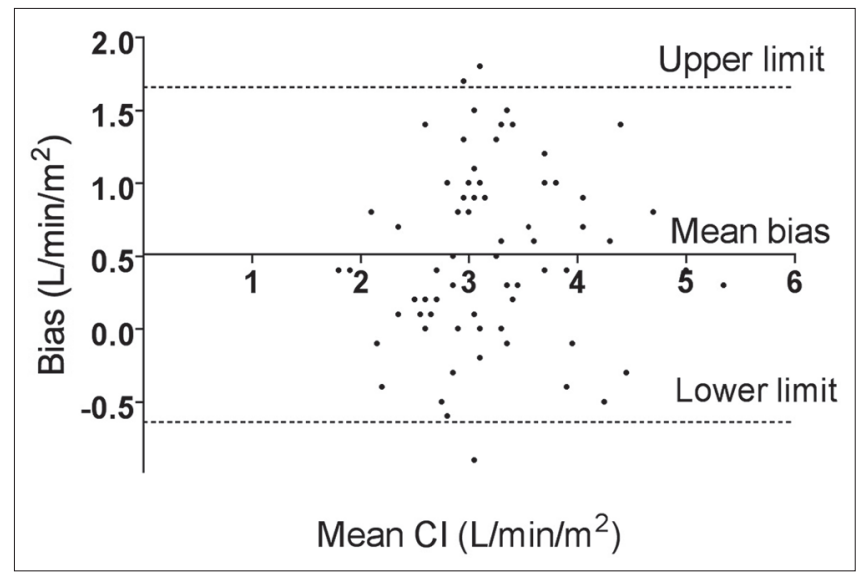

Figure 6: Bland-Altman analysis between transesophageal Doppler and bioreactance in the population of children weighing $>15 \mathrm{~kg}$

by the fact that our patients were all undergoing major abdominal surgery and the room dedicated to bioreactance's electrodes was reduced. Values of CI derived from bioreactance were continuously lower than that from TED even if it does not reach statistical significance. This is consistent with the results of the only 
available bioreactance study performed in pediatrics, which found that the CI measured by bioreactance is lower than the normal range in a large percentage of measurements. ${ }^{[14]}$ In an additional study in piglets submitted to experimental hemorrhage, the same authors found that CI measurements were persistently lower with bioreactance as compared to those obtained with both PAC or pulmonary artery thermodilution. ${ }^{[15,17]}$ The authors concluded that bioreactance was not a suitable method for monitoring the $\mathrm{CI}$ in pediatric hemorrhagic shock. The accuracy should be improved by modification of the internal algorithms by the manufacturer.

We did not assess fluid responsiveness because we limited the purpose of our study to linear comparison of $\mathrm{CI}$ between the two techniques.

Our study presents several limitations. The population was limited to 16 patients but all were monitored by TED and had major abdominal surgery. Another major limitation is our reference technique, namely the TED. Because insertion of central venous and arterial lines were not justified for the care of our patients, we did not use the PAC, nor the transpulmonary thermodilution. A good alternative would have been to use transesophageal echocardiography (TEE) but this requires special probes and training. This must be counterbalanced by the fact that three studies have validated TED versus a goldstandard in children. Tibby et al. have shown that TED provides a clinically accurate estimate of $\mathrm{CO}$ across the entire pediatric age range and is able to follow changes in CO compared to a femoral arterial thermodilution technique. ${ }^{[21]}$ This finding has been recently confirmed by another team comparing TED and transthoracic echocardiography. ${ }^{[22]}$ Tibby et al. also demonstrated that TED used in Intensive Care Unit was able to follow stroke volume changes during a fluid challenge. ${ }^{[9]}$ TED is used daily for pediatric perioperative management in our unit and anesthesiologists are well-trained for its use and interpretation. Moreover, TED is a less aggressive technique and easier to teach and to use than TEE. TED thus represents a clinically relevant comparator.

Bioreactance demonstrated several interesting properties, the major one being its totally noninvasive feature. Even so-called less invasive monitors expose to a risk of complications, although if they appear to be of limited importance for the TED: Minor trauma to the buccal cavity, ${ }^{[23]}$ transient vagal response during probe insertion ${ }^{[24]}$ and bronchial misplacement of the probe. ${ }^{[25]}$ Second, bioreactance is easy to use and requires a very brief tutorial. Measurements are made through 4 pairs of electrodes, which can be placed in the back of the patient, allowing the device to be used during abdominal surgery. Recently, a meta-analysis has confirmed the interest of goal-directed therapy to decrease perioperative risk of pneumonia, pulmonary edema and hospital length of stay. ${ }^{[2]}$ Even though similar data are still lacking in pediatric population, it can be expected that benefits of fluid-guided therapy would be similar. The development of a new noninvasive monitor is of great interest. Its validation and ability to predict fluid responsiveness may need further work.

We demonstrated that bioreactance monitoring was not suitable for pediatric patients. As CI values derived from bioreactance were continuously lower than that from TED it is probable that the accuracy could be improved by modifying the internal algorithm of the bioreactance monitor. Our results need to be confirmed in a larger population and evaluation of the dynamic parameters deserves further research as well. Due to these limitations, bioreactance cannot be recommended to evaluate $\mathrm{CO}$ during major abdominal surgery in pediatric patients.

\section{References}

1. Giglio MT, Marucci M, Testini M, Brienza N. Goal-directed haemodynamic therapy and gastrointestinal complications in major surgery: A meta-analysis of randomized controlled trials. Br J Anaesth 2009;103:637-46.

2. Corcoran T, Rhodes JE, Clarke S, Myles PS, Ho KM. Perioperative fluid management strategies in major surgery: A stratified meta-analysis. Anesth Analg 2012;114:640-51.

3. Abbas SM, Hill AG. Systematic review of the literature for the use of oesophageal Doppler monitor for fluid replacement in major abdominal surgery. Anaesthesia 2008;63:44-51.

4. Sandham JD, Hull RD, Brant RF, Knox L, Pineo GF, Doig CJ, et al. A randomized, controlled trial of the use of pulmonary-artery catheters in high-risk surgical patients. N Engl J Med 2003;348:5-14.

5. Mayer J, Boldt J, Mengistu AM, Röhm KD, Suttner S. Goal-directed intraoperative therapy based on autocalibrated arterial pressure waveform analysis reduces hospital stay in high-risk surgical patients: A randomized, controlled trial. Crit Care 2010;14:R18.

6. Lopes MR, Oliveira MA, Pereira VO, Lemos IP, Auler JO Jr, Michard F. Goal-directed fluid management based on pulse pressure variation monitoring during high-risk surgery: A pilot randomized controlled trial. Crit Care 2007;11:R100.

7. Funk DJ, Moretti EW, Gan T.J. Minimally invasive cardiac output monitoring in the perioperative setting. Anesth Analg 2009;108:887-97.

8. Peyton PJ, Chong SW. Minimally invasive measurement of cardiac output during surgery and critical care: A meta-analysis of accuracy and precision. Anesthesiology 2010;113:1220-35.

9. Tibby SM, Hatherill M, Durward A, Murdoch LA. Are transoesophageal Doppler parameters a reliable guide to paediatric haemodynamic status and fluid management? Intensive Care Med 2001;27:201-5.

10. Brissaud O, Guichoux J, Villega F, Orliaguet G. What non invasive haemodynamic assessment in paediatric intensive care unit in 2009 ? Ann Fr Anesth Reanim 2010;29:233-41.

11. Keren H, Burkhoff D, Squara P. Evaluation of a noninvasive continuous cardiac output monitoring system based on thoracic bioreactance. Am J Physiol Heart Circ Physiol 2007;293:H583-9.

12. Raval NY, Squara P, Cleman M, Yalamanchili K, Winklmaier M, Burkhoff D. 
Multicenter evaluation of noninvasive cardiac output measurement by bioreactance technique. J Clin Monit Comput 2008;22:113-9.

13. Marqué S, Cariou A, Chiche JD, Squara P. Comparison between Flotrac-Vigileo and bioreactance, a totally noninvasive method for cardiac output monitoring. Crit Care 2009;13:R73.

14. Squara P, Rotcajg D, Denjean D, Estagnasie P, Brusset A. Comparison of monitoring performance of Bioreactance vs. pulse contour during lung recruitment maneuvers. Crit Care 2009;13:R125.

15. Ballestero Y, López-Herce J, Urbano J, Solana MJ, Botrán M, Bellón JM, et al. Measurement of cardiac output in children by bioreactance. Pediatr Cardiol 2011;32:469-72.

16. Weisz DE, Jain A, MeNamara PJ, EL-Khuffash A. Non-invasive cardiac output monitoring in neonates using bioreactance: A comparison with echocardiography. Neonatology 2012;102:61-7.

17. Ballestero Y, Urbano J, López-Herce J, Solana MJ, Botrán M, Vinciguerra D, et al. Pulmonary arterial thermodilution, femoral arterial thermodilution and bioreactance cardiac output monitoring in a pediatric hemorrhagic hypovolemic shock model. Resuscitation 2012;83:125-9.

18. Bland JM, Altman DG. Statistical methods for assessing agreement between two methods of clinical measurement. Lancet 1986;1:307-10.

19. Squara P, Cecconi M, Rhodes A, Singer M, Chiche JD. Tracking changes in cardiac output: Methodological considerations for the validation of monitoring devices. Intensive Care Med 2009;35:1801-8.
20. Schober P, Loer SA, Schwarte LA. Perioperative hemodynamic monitoring with transesophageal Doppler technology. Anesth Analg $2009 ; 109: 340-53$

21. Tibby SM, Hatherill M, Murdoch IA. Use of transesophageal Doppler ultrasonography in ventilated pediatric patients: Derivation of cardiac output. Crit Care Med 2000;28:2045-50.

22. Schubert S, Schmitz T, Weiss M, Nagdyman N, Huebler M, Alexi-Meskishvili V, et al. Continuous, non-invasive techniques to determine cardiac output in children after cardiac surgery: Evaluation of transesophageal Doppler and electric velocimetry. J Clin Monit Comput 2008;22:299-307.

23. Singer M, Clarke J, Bennett ED. Continuous hemodynamic monitoring by esophageal Doppler. Crit Care Med 1989;17:447-52.

24. Stawicki SP, Hoff WS, Cipolla J, deQuevedo R. Use of non-invasive esophageal echo-Doppler system in the ICU: A practical experience. J Trauma 2005;59:506-7.

25. Chandan GS, Hull JM. Incorrectly placed oesophageal Doppler probe. Anaesthesia 2004;59:723.

How to cite this article: Dubost C, Bouglé A, Hallynck C, Le Dorze M, Roulleau P, Baujard C, Benhamou D. Comparison of monitoring performance of bioreactance versus esophageal Doppler in pediatric patients. Indian J Crit Care Med 2015;19:3-8. Source of Support: Nil, Conflict of Interest: None declared.

\section{"Quick Response Code" link for full text articles}

The journal issue has a unique new feature for reaching to the journal's website without typing a single letter. Each article on its first page has a "Quick Response Code". Using any mobile or other hand-held device with camera and GPRS/other internet source, one can reach to the full text of that particular article on the journal's website. Start a QR-code reading software (see list of free applications from http://tinyurl.com/ yzlh2tc) and point the camera to the QR-code printed in the journal. It will automatically take you to the HTML full text of that article. One can also use a desktop or laptop with web camera for similar functionality. See http://tinyurl.com/2bw7fn3 or http://tinyurl.com/3ysr3me for the free applications. 